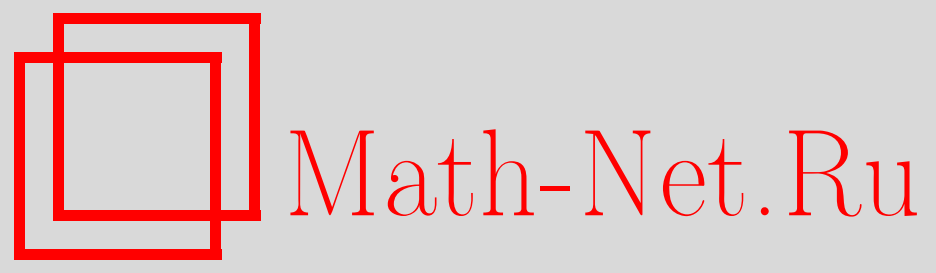

Т. Хуссейн, С. С. Ахтар, Ф. Хан, Классификация локально вращательно-симметричных пространстввремен типа Бианки-І с использованием конформных коллинеаций Риччи, ТМФ, 2017, том 193, номер 1, 133145

DOI: https://doi.org/10.4213/tmf9279

Использование Общероссийского математического портала Math-Net.Ru подразумевает, что вы прочитали и согласны с пользовательским соглашением http://www . mathnet.ru/rus/agreement

Параметры загрузки:

IP: 54.198 .55 .26

26 апреля 2023 г., 16:18:54

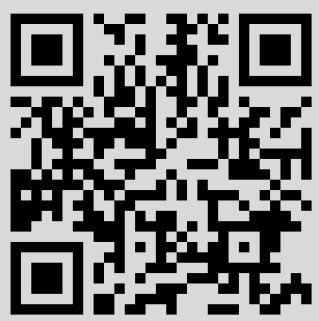




\title{
ФИЗИКА
}

Том 193, № 1

октябрь, 2017

(C) 2017 г.

Т. Хуссейн*, С. С. Ахтар*, Ф. Хан*

\section{КЛАССИФИКАЦИЯ ЛОКАЛЬНО ВРАЩАТЕЛЬНО-СИММЕТРИЧНЫХ ПРОСТРАНСТВ-ВРЕМЕН ТИПА БИАНКИ-І С ИСПОЛЬЗОВАНИЕМ КОНФОРМНЫХ КОЛЛИНЕАЦИЙ РИЧЧИ}

\begin{abstract}
Приведена исчерпывающая классификация локально вращательно-симметричных пространств-времен типа Бианки-I в соответствии с их конформными коллинеациями Риччи. В случае, когда тензор Риччи невырожден, найден общий вид векторного поля, порождающего конформные коллинеации Риччи, удовлетворяющие некоторым условиям интегрируемости. После нахождения решений условий интегрируемости оказалось, что локально вращательно-симметричные пространства-времена типа Бианки-I допускают семи-, десяти-, одиннадцати- или пятнадцатимерную алгебру Ли конформных коллинеаций Риччи в случае невырожденного тензора Риччи. Более того, оказалось, что эти пространства-времена допускают бесконечное количество конформных коллинеаций Риччи, если тензор Риччи вырожден. Приведены несколько примеров локально вращательно-симметричного пространства-времени типа Бианки-I, допускающего нетривиальные конформные коллинеации Риччи и являющегося моделью идеальной жидкости.
\end{abstract}

Ключевые слова: конформные коллинеации Риччи, коллинеации Риччи, пространства-времена типа Бианки-I.

DOI: https://doi.org/10.4213/tmf9279

\section{1. ВВЕДЕНИЕ}

Основной революционной идеей общей теории относительности Эйнштейна являлось предположение о том, что присутствие материи порождает кривизну пространства-времени. Данная идея связала друг с другом геометрию и материю по-

*Department of Mathematics, University of Peshawar, Khyber Pakhtunkhwa, Pakistan. E-mail: tahirhussain@uop.edu.pk 
средством хорошо известных уравнений Эйнштейна (см. книгу [1])

$$
R_{a b}-\frac{R}{2} g_{a b}=G T_{a b},
$$

где через $R_{a b}, T_{a b}$ и $g_{a b}$ обозначены тензоры Риччи, энергии-импульса и метрический тензор соответственно, $R$ - скаляр Риччи, $G$ - гравитационная постоянная. Поиск точных решений уравнений Эйнштейна - важная задача общей теории относительности, открывшей новые перспективы в нашем понимании устройства вселенной. В силу того, что эти уравнения в высшей степени нелинейны, очень сложно найти их точные решения. Поэтому в литературе рассмотрено лишь некоторое относительно малое количество физически интересных решений уравнений Эйнштейна (см. работы [1]-[3]). Все данные решения получены в предположении, что они обладают некоторой симметрией.

Симметрии (коллинеации) суть векторные поля, сохраняющие некоторые интересные геометрические объекты пространства-времени. Среди данных объектов можно отметить метрический тензор, тензоры Римана, энергии-импульса и Риччи. Для того чтобы геометрический объект $\Omega$ сохранялся при коллинеации (векторном поле) $\xi$, потребуем, чтобы производная Ли объекта $\Omega$ была равна нулю вдоль направления $\xi$, а именно (см. книгу [4]):

$$
\mathcal{L}_{\xi} \Omega=0,
$$

где через $\mathcal{L}_{\xi}$ обозначен оператор производной Ли вдоль векторного поля $\xi$. Поле $\xi$ является вектором Киллинга (BK), если в выражении (1.2) $\Omega$ есть метрический тензор $g_{a b}$. Если в (1.2) через $\Omega$ обозначен тензор Риччи $R_{a b}$ или тензор Римана $R_{b c d}^{a}$, тогда соответствующие им симметрии называются коллинеациями Риччи (KP) или коллинеациями, сохраняющими кривизну (KK) [5]. Аналогично можно задать коллинеации, сохраняющие материю (KM), заменяя в формуле (1.2) $\Omega$ на тензор энергии-импульса. ВК являются предметом всесторонних исследований, поскольку с их помощью можно лучше понять решения уравнений Эйнштейна и соответствующие законы сохранения [1], [2]. КР и KK также подробно исследуются в различных работах для того, чтобы достичь лучшего понимания физики пространства-времени в рамках общй теории относительности. Пионер этих исследований Катцин в работе [6] утверждал, что KK следует изучать, поскольку это позволит получить дополнительную информацию о физике пространства-времени, которую нельзя получить с использованием ВК. Всеобъемлющая и заслуживающая внимания книга [4], посвященная KK, опубликована Холлом. В ней также традиционно обсуждаются другие типы симметрий. Поскольку тензор Риччи является следом тензора Римана, КР естественным образом обладают значимостью с геометрической точки зрения [7]-[9]. Важность КР с физической точки зрения также изучена в литературе. Дэвис с соавторами в статьях [10], [11] детально рассмотрел этот вопрос, используя законы сохранения, допустимые в полях материи некоторых типов. Они также предложили несколько интересных приложений своих результатов в релятивистской гидродинамике и физике плазмы. Оливер и Дэвис в работе [12] использовали КР, чтобы получить выражения для законов сохранения в идеальных жидкостях. Некоторые интересные свойства пространств-времен идеальной жидкости исследованы в статье [13]). Помимо всех этих причин важности 
КР с геометрической и физической точек зрения они в том числе полезны при классификации известных точных решений уравнений Эйнштейна, а также при поиске новых точных решений [7], [14]-[18].

Наследованные симметрии представляют особый интерес, поскольку они позволяют более глубоко понять геометрию пространства-времени и важны с точки зрения физического смысла. Данные симметрии применяются при поиске естественной взаимосвязи между геометрией и материей с использованием уравнений Эйнштейна. Одной из хорошо известных наследованных симметрий является конформный вектор Киллинга (KBK), удовлетворяющий соотношению [4]

$$
\mathcal{L}_{\xi} g_{a b}=2 \lambda g_{a b}
$$

где $\lambda$ - гладкая вещественнозначная функция, зависящая от координат пространстввремен, называемая конформным множителем. Если $\lambda=0$, тогда решение уравнения (1.3) является BK, а при $\lambda=$ const оно является гомотетичным вектором (ГВ). $\mathrm{KBK}$ называется специальным KBK, если $\lambda_{; a b}=0$. KBK отображают нулевые геодезические в нулевые геодезические и сохраняют структуру нулевого конуса. Также они порождают интегралы движения вдоль нулевых геодезических для безмассовых частиц. Это свойство связывает конформную симметрию с корректно определенной и имеющей физический смысл сохраняющейся величиной. Влияние конформных симметрий в том числе можно увидеть на кинематическом и динамическом уровнях [19]. Более подробно KBK рассмотрены в статьях [20]-[25].

После метрического тензора $g_{a b}$ важным объектом исследования, играющим значительную роль в понимании геометрической структуры пространства-времени, является тензор Риччи $R_{a b}$. Если в выражении (1.3) вместо $g_{a b}$ использован тензор $R_{a b}$, то $\xi$ называется конформной коллинеацией Риччи (KKP). В этом случае формулу (1.3) можно переписать в явном виде [26], [27]

$$
R_{a b, c} \xi^{c}+R_{b c} \xi_{, a}^{c}+R_{a c} \xi_{, b}^{c}=2 \lambda R_{a b}
$$

При $\lambda=$ const KKP называется гомотетичной коллинеацией Риччи (ГКР), а если $\lambda=0$, то KKР превращаются в КР. Если $\xi$ одновременно представляет собой КВК с конформным множителем $\alpha$ и KKP с конформным множителем $\lambda$, то в таком случае $\alpha$ и $\lambda$ связаны следующим соотношением [26]:

$$
\alpha_{; a b}=-\lambda\left(R_{a b}-\frac{R}{6} g_{a b}\right),
$$

где в правой части член в скобках - хорошо известный тензор Схоутена, который обозначен как $A_{g}$. Важность тензора Схоутена в рамках конформной геометрии обусловлена тем, что он участвует в разложении тензора кривизны Римана $R_{g}$ : $R_{g}=W_{g}+A_{g} \odot g$, где $W_{g}$ - конформно-инвариантный тензор Вейля метрики $g$ и $\odot$ есть произведение Кулкарни-Номидзу.

Множество $C R C(M)$, состоящее из всех KKP метрики пространства-времени, представляющих многообразие $M$, образует векторное пространство, размерность которого может оказаться бесконечной в случае, если тензор Риччи вырожден (т. е. $\left.\operatorname{det} R_{a b}=0\right)$. Однако для невырожденного тензора Риччи $\left(\operatorname{det} R_{a b} \neq 0\right)$ множество $C R C(M)$ образует конечномерную алгебру Ли такую, что $\operatorname{dim} C R C(M) \leqslant 15$. 
Наибольшее значение размерности пространства $C R C(M)$ достигается, если и только если пространство-время является конформно-плоским [26].

В то время как КР и ГКР активно обсуждаются в литературе [28]-[30], KKР вызывают относительно меньший интерес. Лишь для пространства-времени Фридмана-Робертсона-Уокера (ФРУ) и сферически-симметричного статического пространства-времени осуществлена классификация в соответствии с их KKP [16], [26]. Мы надеемся, что классификация пространств-времен в соответствии с конформными коллинеациями тензора Риччи может представлять интерес, и поэтому проводим в настоящей статье исчерпывающую классификацию локально вращательно-симметричных пространств-времен типа Бианки-I с использованием КKР.

Статья организована следующим образом. В разделе 2 мы вкратце описываем пространства-времена типа Бианки и приводим десять связанных уравнений для поиска ККР в случае локально вращательно-симметричных пространств-времен типа Бианки-I. В разделе 3 мы проводим исчерпывающую классификацию этих пространств-времен в соответствии с их KKP, если тензор Риччи невырожден, а в разделе 4 мы решаем уравнения для поиска KKР в случае вырожденного тензора Риччи. В заключительном разделе 5 приводится краткое изложение работы и рассмотрены некоторые физические приложения полученных результатов.

\section{2. УРАВНЕНИЯ ДЛЯ ККР}

Космологические модели типа Бианки-I, Бианки-II, ..., Бианки-IX - это пространственно однородные пространства-времена размерности $1+3$, допускающие группу движений $G_{3}$, действующую на пространственноподобных гиперповерхностях [1], [31]. В литературе данные пространства-времена активно обсуждаются в точки зрения симметрийного подхода. Пространства-времена типа Бианки-І среди них являются наиболее простыми космологическими моделями, для которых группа $G_{3}$ есть абелева группа трансляций трехмерного евклидова пространства. Метрика пространств-времен типа Бианки-I в синхронных координатах имеет вид [1]

$$
d s^{2}=-d t^{2}+h^{2}(t) d x^{2}+k^{2}(t) d y^{2}+l^{2}(t) d z^{2},
$$

где $h(t), k(t)$ и $l(t)$ - функции только от временной координаты. Три основных ВК, допускаемые этой метрикой, выглядят следующим образом:

$$
\xi_{(1)}=\partial_{x}, \quad \xi_{(2)}=\partial_{y}, \quad \xi_{(3)}=\partial_{z} .
$$

Если две из трех метрических функций $h, k$ и $l$ равны, тогда пространство-время типа Бианки-I превращается в важный класс локально вращательно-симметричных (ЛВС) пространств-времен [1], и в этом случае допускается еще один вращательный $\mathrm{BK} z \partial_{y}-y \partial_{z}$. В нашей статье мы полагаем, что в выражении $(2.1) k=l$, и вычисляем ККР для метрики ЛВС-пространства-времени типа Бианки-I. Оставшиеся компоненты тензора Риччи для этой метрики имеют вид

$$
\begin{aligned}
& R_{00}=-\frac{h^{\prime \prime} k+2 h k^{\prime \prime}}{h k}=A(t), \\
& R_{11}=\frac{h h^{\prime \prime} k+2 h h^{\prime} k^{\prime}}{k}=B(t), \\
& R_{22}=R_{33}=\frac{h k k^{\prime \prime}+h^{\prime} k k^{\prime}+h k^{\prime 2}}{h}=C(t),
\end{aligned}
$$


где штрих у метрической функции означает производную по $t$. Скаляр кривизны Риччи $R$ определяется как

$$
R=2\left(\frac{h^{\prime \prime}}{h}+2 \frac{k^{\prime \prime}}{k}\right)+2\left(\frac{k^{\prime 2}}{k^{2}}+\frac{2 h^{\prime} k^{\prime}}{h k}\right) .
$$

Метрический тензор Риччи для ЛВС-пространств-времен типа Бианки-I можно записать посредством выражения

$$
d s_{\mathrm{Ric}}^{2}=R_{a b} d x^{a} d x^{b}=A(t) d t^{2}+B(t) d x^{2}+C(t)\left(d y^{2}+d z^{2}\right),
$$

совпадающего с исходным выражением для метрики ЛВС-пространств-времен типа Бианки-I. Сигнатура записанной выше метрики тензора Риччи является лоренцевой, если $A$ и $B, C$ имеют противоположные знаки, и является положительно или отрицательно определенной, если $A, B$ и $C$ имеют одинаковые знаки. Используя уравнений Эйнштейна (1.1) при $G=1$, мы получаем следующие компоненты тензора энергия-импульса:

$$
\begin{aligned}
& T_{00}=2 \frac{h^{\prime} k^{\prime}}{h k}+\frac{k^{2}}{k^{2}}, \\
& T_{11}=-h^{2}\left(\frac{k^{2}}{k^{2}}+\frac{2 k^{\prime \prime}}{k}\right), \\
& T_{22}=T_{33}=-k^{2}\left(\frac{h^{\prime \prime}}{h}+\frac{k^{\prime \prime}}{k}+\frac{h^{\prime} k^{\prime}}{h k}\right) .
\end{aligned}
$$

Подставляя компоненты тензора Риччи (2.3) в формулу (1.4), приходим к уравнениям для КKР

$$
\begin{aligned}
A^{\prime} \xi^{0}+2 A \xi_{, 0}^{0} & =2 \lambda A, & A \xi_{, 1}^{0}+B \xi_{, 0}^{1} & =0, \\
A \xi_{, 2}^{0}+C \xi_{, 0}^{2} & =0, & A \xi_{, 3}^{0}+C \xi_{, 0}^{3} & =0, \\
B^{\prime} \xi^{0}+2 B \xi_{, 1}^{1} & =2 \lambda B, & B \xi_{, 2}^{1}+C \xi_{, 1}^{2} & =0, \\
B \xi_{, 3}^{1}+C \xi_{, 1}^{3} & =0, & C^{\prime} \xi^{0}+2 C \xi_{, 2}^{2} & =2 \lambda C, \\
C\left(\xi_{, 3}^{2}+\xi_{, 2}^{3}\right) & =0, & C^{\prime} \xi^{0}+2 C \xi_{, 3}^{3} & =2 \lambda C .
\end{aligned}
$$

Данная система содержит десять связанных между собой дифференциальных уравнений в частных производных первого порядка, линейных по конформному вектору $\xi=\left(\xi^{0}, \xi^{1}, \xi^{2}, \xi^{3}\right)$ и по конформному множителю $\lambda$. Решая эту систему, можно найти явный вид для ККР в ЛВС-пространствах-временах типа Бианки-I. В следующих двух разделах мы решаем данные уравнения, рассматривая случаи невырожденного и соответственно вырожденного тензора Риччи, не используя никаких ограничений на вид тензора энергии-импульса $T_{a b}$.

\section{3. ККР В СЛУЧАЕ НЕВЫРОЖДЕННОГО ТЕНЗОРА РИЧЧИ}

В настоящем разделе мы считаем, что тензор Риччи $R_{a b}$ невырожден: $\operatorname{det} R_{a b} \neq 0$. Это означает, что $A, B$ и $C$ не равны нулю. Используя алгебраические преобразования, можно найти общее решение системы (2.7) в терминах некоторых неизвестных 
функций по переменным $t$ и $x$ :

$$
\begin{aligned}
& \xi^{0}=-\frac{C}{A}\left[\frac{y^{2}+z^{2}}{2} P_{t}^{1}(t, x)+z P_{t}^{2}(t, x)+y P_{t}^{3}(t, x)\right]+P^{0}(t, x), \\
& \xi^{1}=-\frac{C}{B}\left[\frac{y^{2}+z^{2}}{2} P_{x}^{1}(t, x)+z P_{x}^{2}(t, x)+y P_{x}^{3}(t, x)\right]+P^{4}(t, x), \\
& \xi^{2}=a_{2} y z+a_{3}\left(\frac{z^{2}-y^{2}}{2}\right)+a_{4} z+y P^{1}(t, x)+P^{3}(t, x), \\
& \xi^{3}=a_{2}\left(\frac{z^{2}-y^{2}}{2}\right)-a_{3} y z-a_{4} y+z P^{1}(t, x)+P^{2}(t, x),
\end{aligned}
$$

где конформный множитель имеет вид

$$
\begin{aligned}
\lambda= & -\frac{C^{\prime}}{2 A}\left[\frac{y^{2}+z^{2}}{2} P_{t}^{1}(t, x)+z P_{t}^{2}(t, x)+y P_{t}^{3}(t, x)\right]+\frac{C^{\prime}}{2 C} P^{0}(t, x)+ \\
& +a_{2} z-a_{3} y+P^{1}(t, x) .
\end{aligned}
$$

Эта система является общим решением уравнений КKР если выполнены следующие условия интегрируемости:

$$
\begin{gathered}
\frac{1}{2}\left(\frac{C^{\prime}}{C}-\frac{B^{\prime}}{B}\right) P_{t}^{1}(t, x)-\frac{A}{B} P_{x x}^{1}(t, x)=0 \\
\frac{1}{2}\left(\frac{C^{\prime}}{C}-\frac{B^{\prime}}{B}\right) P_{t}^{2}(t, x)-\frac{A}{B} P_{x x}^{2}(t, x)-a_{2} \frac{A}{C}=0 \\
\frac{1}{2}\left(\frac{C^{\prime}}{C}-\frac{B^{\prime}}{B}\right) P_{t}^{3}(t, x)-\frac{A}{B} P_{x x}^{3}(t, x)+a_{3} \frac{A}{C}=0 \\
2 C P_{t x}^{1}(t, x)+B\left(\frac{C}{B}\right)^{\prime} P_{x}^{1}(t, x)=0, \quad 2 C P_{t x}^{2}(t, x)+B\left(\frac{C}{B}\right)^{\prime} P_{x}^{2}(t, x)=0, \\
2 C P_{t x}^{3}(t, x)+B\left(\frac{C}{B}\right)^{\prime} P_{x}^{3}(t, x)=0, \quad \frac{C}{A} P_{t t}^{1}(t, x)+\frac{1}{2}\left(\frac{C}{A}\right)^{\prime} P_{t}^{1}(t, x)=0, \\
\frac{C}{A} P_{t t}^{2}(t, x)+\frac{C}{2}\left(\frac{C}{A}\right)^{\prime} P_{t}^{2}(t, x)+a_{2}^{3}(t, x)+\frac{1}{2}\left(\frac{C}{A}\right)^{\prime} P_{t}^{3}(t, x)-a_{3}=0, \\
\frac{1}{2}\left(\frac{B^{\prime}}{B}-\frac{C^{\prime}}{C}\right) P^{0}(t, x)+P_{x}^{4}(t, x)-P^{1}(t, x)=0 \\
\frac{1}{2}\left(\frac{A^{\prime}}{A}-\frac{C^{\prime}}{C}\right) P^{0}(t, x)+P_{t}^{0}(t, x)-P^{1}(t, x)=0 \\
A P_{x}^{0}(t, x)+B P_{t}^{4}(t, x)=0 .
\end{gathered}
$$

Для того чтобы получить окончательные выражения для ККР и соответствующего конформного множителя, требуется решить систему (3.3). Если обозначить $\eta_{i}=\left(\eta_{1}, \eta_{2}, \eta_{3}\right)=\left(\left(y^{2}+z^{2}\right) / 2, z, y\right)$ и $P^{i}=\left(P^{1}, P^{2}, P^{3}\right)$, тогда, применяя правило Эйнштейна суммирования по повторяющимся индексам, мы можем переписать 
систему (3.1) и выражение (3.2) для конформного множителя следующим образом:

$$
\begin{aligned}
\xi^{0} & =-\frac{C}{A} \eta_{i} P_{t}^{i}+P^{0}, \quad \xi^{1}=-\frac{C}{B} \eta_{i} P_{x}^{i}+P^{4}, \\
\xi^{2} & =\left(\eta_{i}\right)_{, 2} P^{i}+a_{2} y z+a_{3}\left(\frac{z^{2}-y^{2}}{2}\right)+a_{4} z \\
\xi^{3} & =\left(\eta_{i}\right)_{3} P^{i}+a_{2}\left(\frac{z^{2}-y^{2}}{2}\right)-a_{3} y z-a_{4} y, \\
\lambda & =-\frac{C^{\prime}}{2 A} \eta_{i} P_{t}^{i}+\frac{C^{\prime}}{2 C} P^{0}+P^{1}+a_{2} z-a_{3} y .
\end{aligned}
$$

Далее, эти условия интегрируемости (3.3) сводятся к условиям

$$
\begin{aligned}
\frac{1}{2}\left(\frac{C^{\prime}}{C}-\frac{B^{\prime}}{B}\right) P_{t}^{i}(t, x)-\frac{A}{B} P_{x x}^{i}(t, x)+k_{i} \frac{A}{C} & =0, \\
2 C P_{t x}^{i}(t, x)+B\left(\frac{C}{B}\right)^{\prime} P_{x}^{i}(t, x) & =0, \\
\frac{C}{A} P_{t t}^{i}(t, x)+\frac{1}{2}\left(\frac{C}{A}\right)^{\prime} P_{t}^{i}(t, x)-k_{i} & =0, \\
\frac{1}{2}\left(\frac{B^{\prime}}{B}-\frac{C^{\prime}}{C}\right) P^{0}(t, x)+P_{x}^{4}(t, x)-P^{1}(t, x) & =0, \\
\frac{1}{2}\left(\frac{A^{\prime}}{A}-\frac{C^{\prime}}{C}\right) P^{0}(t, x)+P_{t}^{0}(t, x)-P^{1}(t, x) & =0, \\
A P_{x}^{0}(t, x)+B P_{t}^{4}(t, x) & =0,
\end{aligned}
$$

в которых $k_{i}=0,-a_{2}, a_{3}$ для $i=1,2,3$ соответственно. Чтобы получить исчерпывающую классификацию при невырожденном тензоре Риччи, мы решаем уравнения (3.5), рассматривая следующие случаи:

ND1) $A^{\prime}=B^{\prime}=C^{\prime}=0$

ND2) $A^{\prime}=C^{\prime}=0, B^{\prime} \neq 0$,

ND3) $B^{\prime}=C^{\prime}=0, A^{\prime} \neq 0$,

ND4) $A^{\prime}=B^{\prime}=0, C^{\prime} \neq 0$,

ND5) $B^{\prime}=0, A^{\prime} \neq 0, C^{\prime} \neq 0$,

ND6) $B^{\prime} \neq 0, A^{\prime}=0, C^{\prime} \neq 0$,

ND7) $B^{\prime} \neq 0, A^{\prime} \neq 0, C^{\prime}=0$,

ND8) $A^{\prime} \neq 0, B^{\prime} \neq 0, C^{\prime} \neq 0$.

Получая решения системы (3.5) во всех перечисленных случаях, мы видим, что ЛВС-пространства-времена типа Бианки-І допускают семи-, десяти-, одиннадцатиили пятнадцатимерную алгебру Ли КKР. Полученные КKР, их конформный множитель и соответствующие ограничения на компоненты тензора Риччи для всех случаев приведены в табл. 1-8 электронной версии настоящей статьи [32]. 


\section{4. ККР В СЛУЧАЕ ВЫРОЖДЕННОГО ТЕНЗОРА РИЧЧИ}

Если тензор Риччи вырожден, т. е. $\operatorname{det} R_{a b}=A B C^{2}=0$, тогда либо $A=B=C=$ 0 , где каждое направление является KKP, либо имеет место одна из следующих ситуаций:

D1) $A \neq 0, B \neq 0, C=0$,

D2) $A=0, B \neq 0, C \neq 0$,

D3) $A \neq 0, B=0, C \neq 0$,

D4) $A \neq 0, B=C=0$,

D5) $A=C=0, B \neq 0$,

D6) $A=B=0, C \neq 0$.

Во всех перечисленных случаях мы решаем систему уравнений (2.7). Если имеет место случай D1, остаются уравнения

$$
\begin{aligned}
A^{\prime} \xi^{0}+2 A \xi_{, 0}^{0} & =2 \lambda A, \\
A \xi_{, 1}^{0}+B \xi_{, 0}^{1} & =0, \\
\xi_{, 2}^{0}=\xi_{, 3}^{0}=\xi_{, 2}^{1}=\xi_{, 3}^{1} & =0, \\
B^{\prime} \xi^{0}+2 B \xi_{, 1}^{1} & =2 \lambda B .
\end{aligned}
$$

Находя решения уравнений (4.2) и (4.3), имеем

$$
\xi^{0}=-\frac{B}{A} f_{t}(t, x)+g(t), \quad \xi^{1}=f_{x}(t, x),
$$

где $f(t, x)$ и $g(t)$ - произвольные функции интегрирования. Подставляя $\xi^{0}(4.5)$ в (4.1), получаем равенство

$$
\lambda=\left(\frac{A^{\prime} B-2 B^{\prime} A}{2 A^{2}}\right) f_{t}(t, x)-\frac{B}{A} f_{t t}(t, x)+\frac{A^{\prime}}{2 A} g(t)+g_{t}(t) .
$$

Используя выражения (4.5) и (4.6), упрощаем формулу (4.4) и приходим к уравнению

$$
\frac{1}{2}\left(\frac{B}{A}\right)^{\prime} f_{t}(t, x)+\frac{B}{A} f_{t t}(t, x)+f_{x x}(t, x)+\left(\frac{B^{\prime}}{2 B}-\frac{A^{\prime}}{2 A}\right) g(t)-g_{t}(t)=0
$$

которое является в высшей степени нелинейным и не решается в общем виде. Однако можно выбрать некоторые функции $f(t, x)$ и $g(t)$ так, чтобы это уравнение выполнялось. Используя эти функции, можно записать две компоненты $\xi^{0}$ и $\xi^{1}$ для $\mathrm{KKP}$, представленные в (4.5), в окончательном виде. Другие две компоненты $\xi^{2}$ и $\xi^{3}$ являются произвольными функциями по переменным $t, x, y$ и $z$. Следовательно, в рассматриваемом случае существует бесконечное число КKР, если имеет место дифференциальное ограничение (4.7).

В остальных пяти случаях нетрудно решить систему для ККР аналогичным образом и увидеть, что в каждом из них существует бесконечное количество удовлетворяющих ей ККР. Эти ККР приведены в табл. 9 в [32]. 


\section{5. КРАТКОЕ ИЗЛОЖЕНИЕ И ОБСУЖДЕНИЕ}

В настоящей статье получена исчерпывающая классификация ЛВС-пространстввремен типа Бианки-I в соответствии с их ККР. Это исследование является расширением нашей предыдущей работы [28], посвященной ГКР в таких пространствахвременах. Считая, что конформный множитель $\lambda=0$, можно провести классификацию ЛВС-пространств-времен типа Бианки-І согласно их КР. Чтобы получить исчерпывающую классификацию ЛВС-пространств-времен типа Бианки-І в соответствии с их KKP, мы рассмотрели случаи невырожденного и вырожденного тензора Риччи. Первым результатом нашей работы является то, что в случае вырожденного тензора Риччи ЛВС-пространства-времена типа Бианки-І допускают бесконечное количество ККР. Более того, наше исследование продемонстрировало, что эти пространства-времена допускают семи-, десяти-, одиннадцати- или пятнадцатимерную алгебру Ли КKР в случае невырожденного тензора Риччи.

Для большинства рассмотренных в статье ситуаций оказалось, что ККР подчиняются некоторым дифференциальным ограничениям, которым удовлетворяют компоненты тензора Риччи. Когда количество KKP равно семи, тогда KKР либо превращаются в ГKP (см. случаи ND2(b) и ND4(b) (здесь и ниже речь идет о случаях, рассмотренных в электронной версии [32]), либо допустима одна собственная KKP вместе с одной собственной ГКР и пятью KP (см. случай ND4(a). В случае ND7(b) имеется десять KKP, среди которых две являются собственными КKР, одна - собственной ГКР и семь являются КР. В случае одиннадцати КKР четыре суть собственные KKP, не имеется ни одной ГКР и семь являются КР (см. случай ND4(b)). Во всех остальных ситуациях, когда тензор Риччи невырожден, мы получили пятнадцать ККР и это количество является максимальным для четырехмерного пространства.

Полагая, что конформный множитель $\lambda=0$ при невырожденном тензоре Риччи, можно видеть, что количество независимых КР, допускаемых в ЛВС-пространствахвременах типа Бианки-I, равно четырем, пяти, шести, семи или десяти. Во всех этих случаях, находя решение дифференциальных условий, которым удовлетворяют компоненты тензора Риччи, мы вывели явный вид для метрик ЛВС-пространств-времен типа Бианки-I, допускающих рассматриваемые КKР. Можно видеть, что упомянутые дифференциальные ограничения в высшей степени нелинейны и их трудно решить в общем виде. Однако с помощью нашей классификации можно понять, что обсуждаемые KKP в принципе существуют. Также можно выбрать некоторые метрические функции $h$ и $k$ таким образом, что дифференциальные условия оказываются верными. Например, полагая $h(t)=t$ и $k(t)=t^{2}$, мы получаем следующую метрику ЛВС-пространства-времени типа Бианки-I:

$$
d s^{2}=-d t^{2}+t^{2} d x^{2}+t^{4}\left(d y^{2}+d z^{2}\right) .
$$

Данная метрика удовлетворяет всем условиям в случае ND5(b) статьи и, таким образом, допускает пятнадцать KKP. Среди этих KKP семь являются KP, а восемь оставшихся - собственными KKР. Скаляр Риччи $R$ для метрики (5.1) имеет вид $R=24 / t^{2}$, а компоненты тензора энергии-импульса суть $T_{00}=8 / t^{2}, T_{11}=-8$ и $T_{22}=T_{33}=-4 t^{2}$, откуда следует, что метрический тензор вещества является невырожденным и лоренцевым. Для остальных случаев можно аналогичным образом найти подобные метрики. 
В процессе получения классификации ККР мы не использовали никаких предположений о виде тензора энергии-импульса $T_{a b}$. Для того чтобы привести пример физического приложения полученных результатов, предположим, что тензор энергии-импульса $T_{a b}$ соответствует модели идеальной жидкости и имеет вид [14]

$$
T_{a b}=(p+\rho) u_{a} u_{b}+p g_{a b},
$$

где $p, \rho$ и $u^{a}$ суть давление, плотность энергии и 4-скорость идеальной жидкости. Мы полагаем, что скорость жидкости $u^{a}=\delta_{0}^{a}$, тогда из формулы (5.2) можно вывести, что $T_{00}=\rho, T_{11}=p h^{2}$ и $T_{22}=T_{33}=p k^{2}$, откуда вытекает следующее выражение для метрического тензора вещества в случае идеальной жидкости:

$$
d s_{\text {Perfect }}^{2}=\rho d t^{2}+p h^{2} d x^{2}+p k^{2}\left(d y^{2}+d z^{2}\right)
$$

данный тензор положительно определен, если $\rho>0$ и $p>0$. Далее, система (2.6) сводится к системе

$$
2 \frac{h^{\prime} k^{\prime}}{h k}+\frac{k^{\prime 2}}{k^{2}}=\rho, \quad \frac{k^{\prime 2}}{k^{2}}+\frac{2 k^{\prime \prime}}{k}=-p, \quad \frac{h^{\prime \prime}}{h}+\frac{k^{\prime \prime}}{k}+\frac{h^{\prime} k^{\prime}}{h k}=-p .
$$

Используя формулы (5.4), можно записать компоненты тензора Риччи (2.3) в виде

$$
A(t)=\frac{3 p+\rho}{2}, \quad B(t)=\frac{h^{2}}{2}(\rho-p), \quad C(t)=\frac{k^{2}}{2}(\rho-p),
$$

а метрический тензор Риччи (2.5) тогда определяется выражением

$$
2 d s_{\mathrm{Ric}}^{2}=(3 p+\rho) d t^{2}+h^{2}(\rho-p) d x^{2}+k^{2}(\rho-p)\left(d y^{2}+d z^{2}\right) .
$$

Метрика (5.6) положительно определена, если $\rho>0,3 p+\rho>0$ и $\rho>|p|$, а именно, если выполняются слабое, сильное и общее энергетические условия. Ниже приведен линейный вид уравнения состояния баротропного газа для $p=p(\rho)$ :

$$
p=(\gamma-1) \rho,
$$

где $\gamma$ - некоторая константа. Значения $\gamma=1,2,4 / 3$ отвечают вселенной, заполненной пылью, твердым веществом и некогерентным излучением соответственно. Значение $\gamma=0$ отвечает вакуумной жидкости. Для идеальной жидкости энергетические условия имеют вид [14]

$$
\rho>0, \quad 0 \leqslant p \leqslant \rho, \quad 0 \leqslant \frac{d p}{d \rho} \leqslant 1 .
$$

В нашем исследовании ККР в ЛВС-пространствах-временах типа Бианки-I состояние идеальной жидкости допускается не во всех случаях, когда тензор Риччи вырожден, а именно, это состояние не допускается в ситуациях D1, D3, D5 и D6. Поэтому в них может выбираться другое состояние вещества. В случае D2 уравнение состояния имеет вид $3 p+\rho=0$ и для него не выполняется сильное энергетическое условие. Также из выражений (2.3) следует, что $h^{\prime \prime} k+2 h k^{\prime \prime}=0$. Можно подобрать функции $h(t)$ и $k(t)$, удовлетворяющие этому равенству, чтобы получить метрику 
ЛВС-пространства-времени типа Бианки-І идеальной жидкости в явном виде. Ниже выписано выражение для одной из таких метрик:

$$
d s^{2}=-d t^{2}+\left(c_{1} t+c_{2}\right)^{2} d x^{2}+\left(c_{3} t+c_{4}\right)^{2}\left[d y^{2}+d z^{2}\right], \quad c_{1} \neq 0, \quad c_{3} \neq 0 .
$$

Компоненты тензора энергии-импульса для данной метрики суть

$$
\begin{gathered}
T_{00}=\frac{c_{3}}{c_{3} t+c_{4}}\left(\frac{2 c_{1}}{c_{1} t+c_{2}}+\frac{c_{3}}{c_{3} t+c_{4}}\right), \quad T_{11}=-c_{3}^{2}\left(\frac{c_{1} t+c_{2}}{c_{3} t+c_{4}}\right)^{2}, \\
T_{22}=T_{33}=-c_{1} c_{3}\left(\frac{c_{3} t+c_{4}}{c_{1} t+c_{2}}\right) .
\end{gathered}
$$

Скаляр Риччи $R$ в рассматриваемом случае принимает вид

$$
R=\frac{2 c_{3}}{c_{3} t+c_{4}}\left(\frac{c_{3}}{c_{3} t+c_{4}}+\frac{2 c_{1}}{c_{1} t+c_{2}}\right) .
$$

Аналогично, из ограничений в случае D4 вытекает условие $p=\rho=A / 2$, являющееся условием для состояния твердого вещества. Тогда система (2.3) приводит к равенствам $h^{\prime \prime} k+2 h^{\prime} k^{\prime}=0$ и $h k k^{\prime \prime}+h^{\prime} k k^{\prime}+h k^{2}=0$. Один из вариантов метрики ЛВС-пространства-времени типа Бианки-I идеальной жидкости имеет вид

$$
d s^{2}=-d t^{2}+e^{2 t} d x^{2}+e^{-t}\left[d y^{2}+d z^{2}\right]
$$

Здесь скаляр Риччи принимает значение $R=3 / 2$. Компоненты тензора энергии импульса даются формулами $T_{00}=-3 / 4, T_{11}=-3 / 4 e^{2 t}$ и $T_{22}=T_{33}=-3 / 4 e^{-t}$. Давление и плотность энергии идеальной жидкости удовлетворяют равенству $p=$ $\rho=-3 / 4$, которое противоречит энергетическим условиям. Также интересно отметить, что соответствующий метрический тензор вещества невырожден и имеет отрицательно определенную сигнатуру.

Если имеет место случай ND1, из условий состояния идеальной жидкости следует, что $3 p+\rho=2, \rho=2 / h^{2}+p$ и $h=k$. Таким образом, метрика ЛВС-пространства-времени типа Бианки-І является хорошо знакомой конформно-плоской метрикой ФРУ при $K=0$. Более подробное обсуждение $\mathrm{KP}$ и $\mathrm{KKP}$ в метрике ФРУ приведено в статье [16]. Аналогичные замечания справедливы и в случае ND3, когда условия состояния идеальной жидкости приводят к равенствам $h=k$ и $p=\rho-2 / h^{2}$. Здесь из системы (2.3) вытекает, что $A=-3 h^{\prime} / h$, где $h$ удовлетворяет дифференциальному ограничению $h h^{\prime \prime}+2 h^{\prime 2}=1$.

Во всех оставшихся ситуациях, когда тензор Риччи невырожден, уравнения идеальной жидкости устанавливают взаимосвязь между давлением $p$ жидкости и плотностью энергии $\rho$, однако из системы (2.3) следуют дифференциальные ограничения на функции $h$ и $k$, имеющие высокую степень нелинейности и поэтому трудноразрешимые. В этом случае можно подобрать некоторые функции $h$ и $k$, для которых данные ограничения выполняются, чтобы получить выражение для метрики ЛВС-пространства-времени типа Бианки-I.

Благодарности. Авторы благодарны рецензентам за ценные замечания, способствующие значительному улучшению статьи. 


\section{Список литературы}

[1] H. Stephani, D. Kramer, M. MacCallum, C. Hoenselaers, E. Herlt, Exact Solutions of Einstein's Field Equations, Cambridge Univ. Press, Cambridge, 2003.

[2] А. З. Петров, Пространства Эйнштейна, Физматгиз, М., 1961.

[3] C. W. Misner, K. S. Thorne, J. A. Wheeler, Gravitation, W. H. Freeman and Co., San Francisco, CA, 1973.

[4] G.S. Hall, Symmetries and Curvature Structure in General Relativity, Lecture Notes in Physics, 46, World Sci., Singapore, 2004.

[5] A. H. Bokhari, A. R. Kashif, "Curvature collineations of some static spherically symmetric space-times", J. Math. Phys., 37:7 (1996), 3498-3504.

[6] G. H. Katzin, J. Levine, "Applications of Lie derivatives to symmetries, geodesic mappings, and first integrals in Riemannian spaces", Colloq. Math., 26 (1972), 21-38.

[7] İ. Yavuz, U. Camci, "Ricci collineations of the Bianchi type II, VIII, and IX space-times", Gen. Relativ. Gravit., 28:6 (1996), 691-700.

[8] U. Camci, H. Baysal, İ. Tarhan, İ. Yilmaz, İ. Yavuz, "Ricci collineations of the bianchi types I and III, and Kantowski-Sachs spacetimes", Internat. J. Modern Phys. D, 10:5 (2001), $751-765$.

[9] U. Camci, İ. Yavuz, "Classifications of Kantowski-Sachs, Bianchi Types I and III spacetimes according to Ricci collineations", Internat. J. Modern Phys. D, 12:1 (2003), 89-100.

[10] W. R. Davis, G.H. Katzin, "Mechanical conservation laws and the physical properties of groups of motions in flat and curved space-times", Amer. J. Phys., 30:10 (1962), 750-764.

[11] W. R. Davis, L.H. Green, L.K. Norris, "Relativistic matter fields admitting Ricci collineations and related conservation laws", Nuovo Cimento B, 34:2 (1976), 256-280.

[12] D. R. Oliver Jr., W. R. Davis, "Perfect fluids and symmetry mappings leading to conservation laws", J. Math. Phys., 17:10 (1976), 1790-1792.

[13] M. Tsamparlis, D. P. Mason, "Ricci collineation vectors in fluid space-times", J. Math. Phys., 31:7 (1990), 1707-1722.

[14] U. Camci, İ. Türkyilmaz, "Ricci collineations in perfect fluid Bianchi V spacetime", Gen. Relativ. Gravit., 36:9 (2004), 2005-2019.

[15] G. Contreras, L. A. Nùñez, U. Percoco, "Ricci collineations for non-degenerate, diagonal and spherically symmetric Ricci tensors", Gen. Relativ. Gravit., 32:2 (2000), 285-294.

[16] U. Camci, A. Barnes, "Ricci collineations in Friedmann-Robertson-Walker spacetimes", Class. Quantum Grav., 19:2 (2002), 393-404.

[17] A. H. Bokhari, "Ricci tensor with six collineations", Internat. J. Theor. Phys., 31:12 (1992), 2091-2094.

[18] J. Llosa, "Collineations of a symmetric 2-covariant tensor: Ricci collineations", J. Math. Phys., 54:7 (2013), 072501, 13 pp.

[19] M. Tsamparlis, A. Paliathanasis, L. Karpathopoulos, "Exact solutions of Bianchi I spacetimes which admit conformal Killing vectors", Gen. Relativ. Gravit., 47:2 (2015), 15, 21 pp.

[20] S. Moopanar, S. D. Maharaj, "Conformal symmetries of spherical spacetimes", Internat. J. Theor. Phys., 49:8 (2010), 1878-1885.

[21] S. Moopanar, S. D. Maharaj, "Relativistic shear-free fluids with symmetry", J. Eng. Math., 82 (2013), 125-131.

[22] K. L. Duggal, R. Sharma, "Conformal Killing vector fields on spacetime solutions of Einstein's equations and initial data", Nonlinear Anal., 63:5-7 (2005), e447-e454.

[23] R. Maartens, S. D. Maharaj, B. O. J Tupper, "General solution and classification of conformal motions in static spherical spacetimes.", Class. Quantum Grav., 12:10 (1995), $2577-2586$.

[24] S. Khan, T. Hussain, A. H. Bokhari, G. A. Khan, "Conformal Killing vectors in LRS Bianchi type V spacetimes", Commun. Theor. Phys., 65:3 (2016), 315-320. 
[25] S. Khan, T. Hussain, A. H. Bokhari, G. A. Khan, "Conformal Killing vectors of plane symmetric four dimensional lorentzian manifolds", Eur. Phys. J. C, 75 (2015), 523, 9 pp.

[26] U. Camci, A. Qadir, K. Saifullah, "Conformal Ricci collineations of static spherically symmetric spacetimes", Commun. Theor. Phys., 49:6 (2008), 1527-1532.

[27] M. Tsamparlis, P. S. Apostolopoulos, "Ricci and matter collineations of locally rotationally symmetric space-times", Gen. Relat. Gravit., 36:1 (2004), 47-69.

[28] T. Hussain, S. S. Akhtar, S. Khan, "Ricci inheritance collineation in Bianchi type I spacetimes", Eur. Phys. J. Plus, 130 (2015), 44.

[29] T. Hussain, S. S. Akhtar, A. H. Bokhari, S. Khan, "Ricci inheritance collineations in Bianchi type II spacetime", Modern Phys. Lett. A, 31:17 (2016), 1650102, 16 pp.

[30] T. Hussain, A. Musharaf, S. Khan, "Ricci inheritance collineations in Kantowski-Sachs spacetimes", Internat. J. Geom. Meth. Modern Phys., 13:5 (2016), 1650057, 14 pp.

[31] R. M. Wald, General Relativity, Chicago Univ. Press, Chicago, IL, 1984.

[32] T. Hussain, S.S. Akhtar, S. Khan, "Classification of LRS Bianchi type I spacetimes via conformal Ricci collineations", arXiv: 1701.06900.

Поступила в редакцию 26.09.2016, после доработки 16.12.2016 